\title{
Social Aging Problem and Pension Market Based on Data Analysis: A Comparison of State Quo Between China and Japan
}

\author{
Yixin Zhang ${ }^{1}$ \\ ${ }^{1}$ Xi'an Gaoxin No.1 High School, Xi'an, Shaanxi, 710075, China \\ *Corresponding author. Email: shilishuang@cas-harbour.org
}

\begin{abstract}
Within Asia, Japan is a typical aging country. In 1970, Japan started to become an aging society, and then the aging process continued to deepen. Around 2010, there appeared a severe social situation of negative natural population growth appeared in Japan. Similar to Japan, as the largest developing country in the world, China also entered the aging society process around 2000, and its aging population is growing rapidly. As typical aging countries, China and Japan are both located in the cultural area of East Asia. There are some similarities between the aging social backgrounds of China and Japan, which is of certain significance for comparative study. At the same time, the aging process of Japan is about 30 years faster than that of China, which means that Japan has more experience in dealing with the aging problem. Therefore, this paper analyzed and discussed the inspiration of Japan's solutions to the aging problem from different perspectives to China. Eventually, through a series of theoretical comparative studies, the author tried to clarify the key role of the pension market in both China and Japan. It turned out that China's pension market has unlimited development potential and commercial value.
\end{abstract}

Keywords: Social aging problem, comparative analysis, China and Japan, pension market, data analysis

\section{INTRODUCTION}

According to the classification of population types and the definition of aging society standards by the International Population Organization, generally speaking, within a country or region, if more than $10 \%$ of the population is over 60 years old, or more than $7 \%$ of the population is over 65 years old, the country or region can be determined as an aging society. Based on this, China entered the aging society around 2000, and its aging process increased rapidly in the primary stage. There are two main reasons for this phenomenon: Firstly, beginning in 1978, the Chinese government launched the one-child policy, which in a short period of time is to effectively controlled the new-born population growth. As a result, around 2010, the net natural population growth appears negative rate. Secondly, after the reform and opening up, China's rapid development in science and technology, economy, medical treatment and other aspects. At the same time, the general improvement of people's living standard has a direct impact on the extension of life expectancy of Chinese people's living standard has a direct impact on the extension of life expectancy of Chinese people, namely from 65 to 73 years old [1]. Similarly, Japan has entered and deepened an aging society as early as the 1970s. Based on nearly 50 years' experience in dealing with aging, the Japanese government came up with "the white paper aging society" in 2018. Its ageing policy improved from the aspect such as social pension, thus increasing the income of the elderly population. Besides, the old began to return to the labor market. What is more, the nursing of the elder people developed. It can be seen that Japan has made some achievements in dealing with the issue of aging, and it has become a model for other countries and regions to learn from [2]. This article focuses on pension policies and the pension market and compares the similarities and differences between China and Japan in dealing with aging issues. In addition, this paper analyzes the aging process in Japan, and the experience that China can learn from Japan in aging. It is hoped that this research can arouse the public's attention to an aging society, provide feasible suggestions for alleviating the pressure of aging on society, and make a more reasonable development for China's pension market on this basis. 


\section{PROBLEMS AND SOLUTIONS IN JAPAN}

From the perspective of Japan's aging situation, the aging population will mainly bring about the following problems: the reduction of labor force leads to a decline in productivity, and the pensions of retirees are negatively affected. The level of investment and purchasing power have dropped severely. Especially from the perspective of the financial industry, the following three main problems have emerged: Firstly, the financial investment of ordinary households has plummeted; Secondly, the financial and insurance intermediary business has fallen sharply and has been restricted; thirdly, the government's relevant policies on the central bank have been restricted [4].

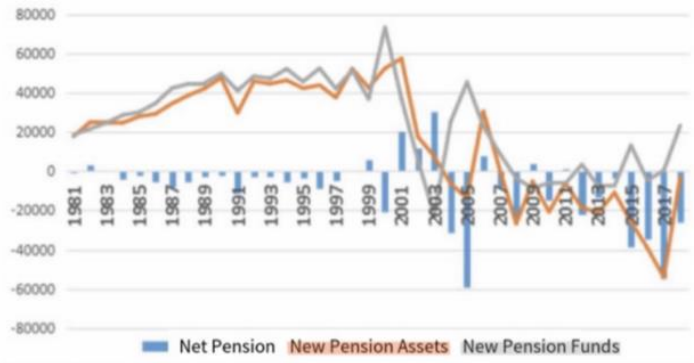

Figure 1. Pension Fund Flow Chart of Japan (19812018). Source: Bank of Japan

In 2015, the then Prime Minister of Japan, Shinzo Abe, proposed two practical solutions: First, improve production technology and create a more advanced production environment. Companies are required to improve the comfort of their employees and fully disclose work information to women and the elderly, thus greatly enhancing the attractiveness of the Japanese labor market; secondly, while introducing policies to promote the development of the elderly care industry, it is also for the aging population. It provides a good opportunity to return to the labor market and solves some social problems caused by retired elderly people. In addition, he also proposed to encourage families to have children from the perspective of population growth to alleviate population aging [5].

\section{JAPAN'S SPECIFIC EXPERIENCE FOR CHINA}

\subsection{Technology}

There is no doubt that Japan's industrialization and modernization in the pension market is earlier than China's. At the same time, due to the serious problems caused by the aging society on the domestic market earlier, the Japanese government encourages and promotes the development of scientific and technological forces in the elderly care market, among which the most remarkable achievement is the nursing robot in the elderly care industry. As mentioned in previous part, the increasing aging has caused the demand for the elderly care industry to far exceed the labor supply, and the income and treatment of nursing workers are not enough to attract a large number of new workers. By the way, some caregivers work very difficult tasks, such as turning over old people who can't take care of themselves. In order to solve such problem, the Japanese government first improved the work welfare of nursing staff, and then vigorously promoted the research and development of nursing robots, and put them into the market and application. Facts have proved that nursing robots not only effectively help nursing workers to reduce the pressure of work content, but also meet the demand for nursing workers in a certain number of elderly market [6]. The power of science and technology eventually promotes the formation of a relatively mature integrated market model in the pension industry, and effectively alleviates the social pressure of short supply.In addition, Japan Kawasaki Care Service successfully implemented a set of e-pension service management mode through a specific APP, which significantly improved the quality and work efficiency of the institution's old-age service [8], and more effectively proved the feasibility of e-pension in the market.

At present, China's aging problem is getting more and more serious, and is facing a state of serious imbalance between the supply and demand of elderly care services. Meanwhile, the development of scientific and technological innovation in the pension market is particularly important, because it can reduce the pressure on pension service staff and reduce the cost of pension services. In addition, it can also achieve more convenient and systematic service management and improve the living standards of the elderly [7].

\subsection{Labor market}

In addition to the emerging geriatric care industry, aging itself is putting enormous pressure on the domestic labor market. Faced with this problem, the Japanese government has put forward two feasible policies: Firstly, gradually postpone the legal retirement age. It is reported that Japan will reach the standard of 65 years old as the legal retirement age in 2025. Secondly, vigorously support the development of the "Silver Industry", encourage capable aging population to return to the labor market, or choose diversified pension methods and bring positive benefits to the market. At present, China is drawing lessons from the experience of Japan, gradually adjusting and postponing the legal retirement age while vigorously developing the "Silver Industry", and has achieved certain results [9].

\subsection{Pension market model}


Japan's pension market has been developing since the early 1970s. By the end of last century, Japan had formed a relatively perfect, mature and appropriate pension market model, that is, mainly family care pension and pension institutions, supplemented by tourism pension.It showed that Japan had formed a relatively sound industry system. And, it is worth to mention that family care penion reflects the influence of Asian culture on society, which values family and filial piety. Additionally, with the development of science and technology industry, the facilities of pension institutions are becoming more and more advanced. Therefore, in Japan, many elderly people would choose suitable pension institutions according to their own conditions. In addition, the diversification of the pension market model in Japan is also reflected in that, according to the market demand of the elderly with different consumption capacity and social or cultural factors in different aspects, projects such as family travel and tour groups for the elderly are customized [10].

At the stage of developing, China's pension market could learned a lot from Japan's pattern. Firstly, the pension market investors in China are supposed to come up with a "New Concept" pension market pattern according to Chinese unique social culture, customs and so on, so that the to be more suitable for China's own situation endowment patterns (because of the similarity on culture and the neighbor relationship between China and Japan,_-Japan's "family-oriented" pension model is worth learning from). Secondly, investors should focus on the innovation and progress of science and technology industry in the pension market field, and provide practical help to develop the pension institutions or elderly care projects. For example, it is needed to design such beds that can help the elderly without the ability to take care of themselves to turn over, so as to assist nursing workers to do better cleaning services for the elderly. Thirdly, consumers in the pension market would be classified according to their consumption power or family economic conditions, and different levels of pension services are provided in a targeted way, such as high-end nursing homes and social nursing homes. Objectively, China's pension market is developing, and it is in the stage of urgent need to refine the pension service industry chain and launch more targeted pension projects to meet different market needs; and it will have huge development potential and commercial interests in the future. In recent years, many European pension service giants (such as France's Orbault) have said that they have seen commercial interests and good development opportunities in China's pension market, and many foreign enterprises also have chosen to enter the Chinese pension market [11]. In addition, China is the only country in the world with an aging population of more than 100 million and the aging degree is constantly deepening. There is no doubt that the development of China's pension market is considerable. Therefore, China's pension market will continue to accelerate development and gradually be refined under the impetus of various commercial forces, which will show great development potential and commercial value.

\section{CONCLUSION AND SUGGESTIONS}

This article analyzes the differences between China and Japan in the aging process and coping styles. And through Japan's experience in actively responding to the aging problem, combined with the similarities and differences of the pension environment of the two countries, it also provides a reference for the development of China's pension market.

According to the research of this article, the author has the following results: At present, Japan has formed a relatively complete elderly care service system. Based on the similarity between China's aging process and the situation in Japan at the end of the last century, it can be inferred that China's pension is also likely to face or is facing the situation that has already occurred in Japan. At the same time, the aging of the population will also cause a series of financial problems. Judging from Japan's effective experience, because of its sound industrial structure, Japan can better cope with an aging society, and China's industrial structure needs to be further improved. In summary, the author puts forward the following suggestions: Firstly, there should be financial insurance products tailored to the actual needs of the elderly; Secondly, there should be professional and systematic management of pension fund investment to avoid risks and attract investors [12]; Thirdly, there should be policies to encourage and guarantee the re-employment of the elderly. Fourth, there should be policies to guide the development of the elderly care market, such as providing social welfare subsidies to nursing homes to improve the welfare and professional quality of nursing staff. In addition, the Chinese pension market should learn from the active measures taken by Japan and even European and American countries to effectively deal with aging. For the Chinese pension market with great development prospects and investment value, the government should encourage domestic investors to invest, manage the market model, and actively accept experienced foreign companies to join. It is believed that with the joint efforts of all parties, the Chinese pension market will develop actively and create considerable commercial value.

\section{REFERENCES}

[1] X. Peng. China's aging under the changing population pattern. Entrepreneur Daily, 2018-05-28 (A02).

[2] Y. Ding. The latest situation, social impact and related social policies of Japan , s 
aging-Interpretation of the 2018 edition of the White Paper on Aging Society. Japanese Studies, 2019(01): 27-37.

[3] X. Shi. Is the impact of aging on Chinese and Japanese societies the same? A comparative study based on the population data of China and Japan. Journal of Guizhou University of Finance and Economics, 2017(02): 21-30.

[4] X. Chen. Looking at the changes in Japan's financial structure under the background of population aging from the flow of funds table. Western Finance, 2020(07): 33-37.

[5] T. Feng. The new development of Japan's aging policy and its enlightenment to China. Population and Economy, 2019(04):79-93.

[6] L. Ping. The Japanese government supports the application of scientific and technological innovation in the elderly care sector-from the perspective of alleviating price distortions. Northeast Asia Journal, 2016(02): 22-27.

[7] P. Guoi. The application of electronic management in Japan's elderly care market-Taking Japan's Kawamoto Care Service Office as an example. Operation and Management, 2013(12): 27-30.

[8] Y. Zhang. Research on the Problems and Countermeasures of the Elderly Care in Science and Technology under the Background of China's Aging. Hefei University of Technology, 2016.

[9] T. Jiang. The impact of population aging on China's labor market and its countermeasures. Macroeconomic Research, 2019(12): 148-159.

[10] J. Dai. Research on the business model of high-end pension industry. East China University of Science and Technology, 2018.

[11] P. Zhu, X. Liu. Foreign giants are competing to deploy 22 trillion Chinese pension market. China Foreign Investment, 2018(03): 54-55.

[12]Sun Min. Population aging and China's capital market development. East China Normal University, 2016. 